\title{
Agent-based Modeling of Social Behavioral Challenges in Transportation
}

\author{
Samar El-Amine ${ }^{a}$, Stephane Galland ${ }^{a}$, Abderraffiaa Koukam ${ }^{\mathrm{a}}$ \\ ${ }^{a}$ LE21, Univ. Bourgogne Franche-Comte, UTBM, 90010 Belfort Cedex, France
}

\begin{abstract}
Agent-Based modeling has been around us for reasonably some time now and has thus developed a vital factor for performing prediction-based scheduling, such as the transportation models for municipal cities. This paper assumes the important understanding of the agent-based modelling and imitation and its application to the transportation models while deliberating the possibility of its applications and advantages too. The paper then presents the ideas recognised to the social behaviours in combination with the agent-based modelling procedures useful so far. The literature review showed in lieu of this work has caused in agreement with the fact that the applicability of agent-based modelling is by far superior than ever due to the ever-improving computing speeds and abilities, while the understanding of complex human behaviour will endure to be a challenge for imitations and mechanisation methods developed so far.
\end{abstract}

Keywords: Agent-Based Modeling (ABM); ABM Transportation Model; Social Behaviors

\section{Introduction}

In agent-based simulation, active objects, recognized as agents, must be recognized and their behaviour is defined. Connections between the agents in the system are established, environmental variables are set, and simulations are run (Samuelson \& Macal 2006). The worldwide dynamics of the system then arise from the connections of the individual activities and manners.

Agent Based Modelling and Simulation (ABMS) is a modelling method for aping the activities and connections of autonomous entities, with a vision of evaluating their impacts on the system all together. ABMS can be understood as a system of independent agents that follow rubrics of communication. Repetitive, competitive connections between agents are major topographies of ABMS, which depend on the power of computers to search dynamics out of the spread of pure mathematical approaches. In a traditional distinct occasion simulation, objects follow a sequence of procedures, which are well-defined from the top-down system viewpoint (Samuelson \& Macal 2006). In contrast, ABMS describes the local behaviour instructions, usually simple, of each object from a bottom-up viewpoint. In agreement, simulation-modelling results disclose the developing behaviours of a system as complete, based on the behaviour formations of the fundamental objects (Bonabesau 2002). The main origins of ABMS are in modelling human, social, organizational behaviour and individual decision-making

Agent Based Modelling and Simulation (ABMS) has been

${ }^{*}$ Corresponding author.

E-mail: samar@sanda.be

(C) 2011 International Association for Sharing Knowledge and Sustainability.

DOI: $10.5383 / J U S P N .10 .01 .005$ mostly related over a series of panels by both specialists and experts. Cases of these panels include nature, science, business, financial science, computer simulation, sociologies, political science, strategy, and military studies. Information and usages of ABMS continue to grow and gather through fast and intuitive, innovative work (Samuelson \& Macal 2006). ABMS has been linked to a wide scope of spaces in transportation (Macal \& North 2006). These applications fundamentally fall into two procedural ideal models: individual-based models that analyse individual transportation-related movements and behaviour, and computational strategies that analyse a community oriented and receptive transportation outline that displays insight by representing a gathering of sovereign basic decision-making of subsystem materials called agents.

The prior is decisively identified with the models for travellers' movements while the latter is usually examined as a computational plan in a distributed artificial intelligence (DAI) outline, or a complex adaptive system (CAS), which is a skilled procedure for imitating dynamic complex outlines to watch new performance. In former studies, it is simple to see transportation studies crossing the limit of the two classes yet examined with the same (or relative) term, agent based, hence stimulating theoretical dismay (Samuelson \& Macal 2006). The objective of this paper is to check the chronicled viewpoints and the continuous progressions of ABMS in the interdisciplinary transportation areas, shortening and clearing up the allowance and key abilities of past agent based reviews and to disclose vision into future possible research.

Another logical attention of this base work is to report a check effort that endeavours to set up the linking between the traditional and ABMS-based sequence decision models. This 
effort plans to response a logical request: Because both recognized econometric models and ABMS are believable in displaying people's route choice decision behaviours, there as far as anyone recognises exist certain circumstances and settings at which both representing ideal replicas show virtually identical effects (Macal \& North 2006). This request, as a vital pace toward a bigger understanding of the traditional econometric technique and ABMS-based procedures, reveals vision into the way ahead for the progression of an all-inclusive modelling framework.cuments supplied by the author(s). To ensure publication quality and uniformity, the following requirements have been prepared to assist authors in preparing papers for the Journal and the Conference. If these requirements are not followed, papers will be returned for revision and re-submittal. The resulting time delay could cause rejection of the paper because of publication deadlines for the Journal Publication or Conference Proceedings.

\section{Fundamental Concepts}

ABMS is a modelling method for rebuilding the activities and infrastructures of self-ruling people, with a view to assessing their significances for the outline in general. A basic thought of ABMS is that many phenomena, even complex ones, can be understood as outlines of independent agents that take after creeds of communication (Helbing n.d.). Repetitive, aggressive associations between experts are important basics of ABMS, which depend on the energy of computers to examine flow out of the time of spotless numerical strategies. In a conservative distinct occasion re-enactment, basics take after a sequence of events, which are considered opening from the top down opinion (Bonabesau 2002). Amusingly, ABMS symbolises the neighbourhood conduct rubrics, normally simple, of every substance from a bottom-up opinion. In considering, simulation consequences expose the developing put into practise of an outline in general, in light of the behaviour preparations of the hidden rudiments. The principle basics of ABMS are in representing human social and authoritative behaviour and individual autonomy.

There is no general meaning of the word agent, as it could refer to numerous parts when studying characteristic goals in numerous ideal models. Some may reflect any sort of renowned parts of a suite (e.g., model, outline, or subsystem), or any type of autonomous component (e.g., association, secure, or distinctive individuals), to be an agent (Epstein 2009). The agent is modified to respond to different authorities and the computational disorder in which it is situated, with a behaviour rule going from primeval response selections to multifaceted versatile.

\section{The Needs and Advantages of Agent-Based}

\section{Simulation for Individual Mobility Simulation}

The ABMS approach permits one to speak to and inspect a complicated matter (e.g., system dynamics) past the variety of mathematical or traditional imitation tackles. Developments in database revolution (authorising a better level of granularity) and computational power permit one to process widespread scale microsimulation models that would not have been imaginable before. This component of ABMS has added to the arena of computer re-enactment by giving additional worldview to the regeneration of complex outlines with numerous relations between the materials of the system
(Epstein 2009). In microsimulations, the construction is seen as increasing from the infrastructures between the people, while in microsimulations, the preparation of people is seen as a construction that can be represented by various issues.

Professional based regenerations are suitable not exclusively to reproduce links between various people. They allow one to start off with the clear drive of verbal argumentation and to choose the consequences of various conjectures. From this point of opinion, computer imitation can give a thoughtful formal structure and instructive device. Other positive mechanisms of agent based modelling contain particularity, awesome flexibility, extensive sadness, and the likelihood to implement them in a parallelized manner (Helbing n.d.). Agent based models can be combined well with diverse kinds of models. One can without much of bounce couple professional based models with range models, for example, liquid dynamic models or gas-motor. Such a method is, for example, used to replicate the clearance of individuals in circumstances where toxic gas binges in the earth. A similar approach would be linked, when natural, climate, or atmosphere reproductions would be combined with replicas of human reaction to the particular outside circumstances (Epstein \& Axtell 2007). At last, agent based simulations are suitable for point by point philosophy testing, i.e. for the examination of the results of exbet conjectures with respect to the relations of agents. In so far, one could say that they can seal in as a kind of intensifying glass or telescope, which might be used to understand our world better. It is commonly no issue to smear methods from figures and econometrics to associate results with actual information.

\section{Diagrammatic Representation of Agent-based Model}

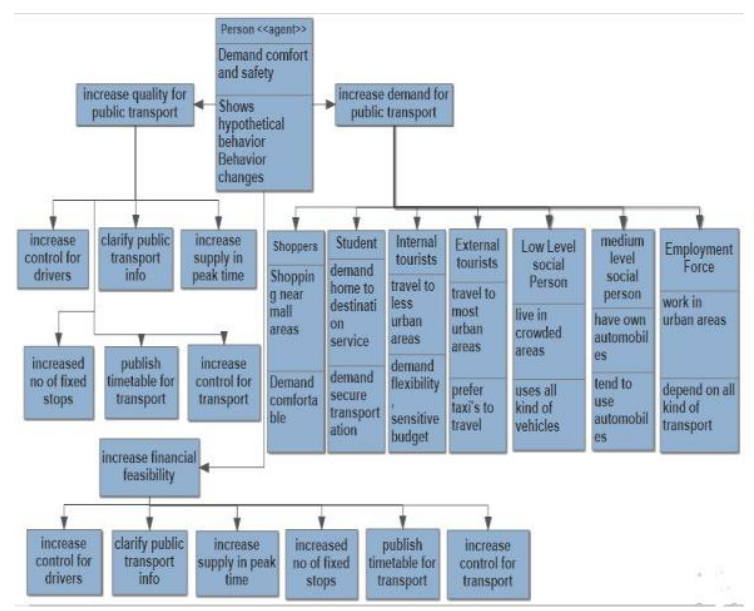

Figure 1: Improved quality of public transportation for agents' demands differences.

The agent is responsible for receiving and producing the transport details. The person can be shopper, student, internal tourist or any external tourist. All of these classes of a person have their own properties and their own behaviours.

Shoppers: The shoppers are referred as people who go to the mall for shopping. They can also be the workers of the mall, so they can have scheduled rides for them. They have two main categories. First they can be active users, or they can be the 
seasonal users. The active users may use the transport on daily basis, and the seasonal users may use it occasionally.

Students: For students, the main concern is the scheduled timing. Every student has his/her scheduled timing to get a trip. Students avails the trip on their time to attend their institutes or to return from the institutes. They also have the fixed destinations to travel. Unlike others, the students are required to be picked and dropped at the fixed destinations all the time. They students always have less financial capabilities, so their price factor is always sensitive.

Secondly students need secure transportation tools at all times. They use either the buses, or their parent's automobiles to travel.

Internal tourists: By internal tourists are local residents of a certain area, but they always plan tours in their own city. They are mostly active on off days towards the less urban sports. They need a reliable transport, so they are sensitive in their selection of transportation since they are aware of the local traffic conditions. They prefer public transportation when travelling to city centers and during rush hours; however, they prefer private transportation in all other cases.

External tourists: External tourists mean people from abroad, who are on a visit to the city. The external tourists are not always aware of the routes of the city, so they usually use maps to travel through the city. They do not know how to find a specific space; they are unaware of the transportation system of the place, so they are relatively not price sensitive. They have flexibility in choosing the place and time. They prefer using taxis or ride-sharing to travel around because of comfort.

Low Social level People: The low social level people are those who live in crowded areas. They are low on resources, so they use the buses or the motorcycles for their transport mean. They can't afford the expensive taxis and don't typically ask for specific transport. They use all types of transport without any hesitation.

Medium Social level People: The medium level people have their own means of transport usually. They care about the price and quality for the transport. They prefer using their own vehicles.

Employment force: It refers to people work in the urban areas. They use the scheduled rides mostly. By scheduled rides mean they usually have the same timings to travel back and forth.

For this category of travellers, parking is the main problem. Even though they have their own means of transportation, but because of the challenge of parking, they don't always use their own vehicle for travelling. They depend on all kinds of transportation depending on the time, day of the week and destination.

OBJECTIVES: There are some objectives, which are to be achieved by the agents in the region:

- The number of fixed stops and the destination should be increased to increase usage of public transport.

- Public transport should be more clarified to the people so that more and more people may get interested in using the public transport system, as it is a better and cheaper option to travel.
- There should be a fix timetable for the public transport, and this timetable should be easily available, so that people can know when to avail their suitable transport.

- During peak hours, the supply of the public transport should be increased so there is no shortage of travel space.

Another objective is the financial feasibility of the public transport. To increase the financial feasibility, the following steps are required to be taken:

- Increase the fuel price, so that the transport mean can earn more by loading passengers, and then they can fulfil the above mentions needs.

- Certain traveller discounts should be made available, such as for the handicapped, senior citizens and students.

- The imported cars should be charged more custom to increase the financial support of the government.

- The public transport prices should be under the control of the government. The government should take over the public transport, and should be assigned the responsibility to handle the public transport and its budget.

- $\quad$ The parking prices should be increase, so that the private transport should pay more parking fee to park their vehicles. The people using their own vehicles should also pay much to avail the parking services at the public places.

- Taxes on private car ownership should also be increase to encourage people to use the public transport.

\section{Subject-area scope}

Applied ABMS is efficiently being applied in many territories. Cases of usage include demonstration of : team working, organizational behaviour and psychology, supply chain management and logistics, social networks, consumer behaviour, distributed computing, environmental study and transportation management. In these applications, the organization of plotting is imitated by catching the behaviour of discrete agents and their interconnections. Operator based representing devices can be applied to test how variations in individual practices will affect developing general conduct of framework (Epstein \& Axtell 2007). ABMS has additionally been linked to diverse spaces in social and society reflects, including populace development, the spread of plagues, organic applications, human progress progression, and armed applications. Macal and North have branded these ABMS applications into two taxonomies;

- Small, delicate, moderate models: Simple models depend on a preparation of admired beliefs, envisioned to latch just the most distinguished components of an outline. These are probing electronic labs in which a widespread variety of beliefs can be altered over uncountable imitations.

- $\quad$ Large-scale choice helpful networks: Decision boost models have a propensity of significant applications, envisioned to answer a wide possibility of policyframework queries. These models are documented by including honest information and having approved some level of endorsement testing to set up rationality in their outcomes. A brief description of these applications has been précised with this. 
Table 1: Agent-Based Modelling Applications

\begin{tabular}{|c|c|}
\hline Functional Area & Applications \\
\hline $\begin{array}{l}\text { Business and } \\
\text { Organizations }\end{array}$ & $\begin{array}{ll}- & \text { Manufacturing processes } \\
- & \text { Supply chains } \\
\text { - } & \text { Customer markets } \\
\text { - } & \text { Insurance Industry }\end{array}$ \\
\hline Economics & $\begin{array}{l}\text { Artificial monetary markets } \\
\text { Trade networks }\end{array}$ \\
\hline Infrastructure & $\begin{array}{l}\text { Electric power markets } \\
\text { Transport } \\
\text { Hydrogen infrastructure }\end{array}$ \\
\hline Crowds & $\begin{array}{l}\text { Pedestrian movement } \\
\text { Evacuation modelling }\end{array}$ \\
\hline Culture & $\begin{array}{l}\text { Ancient civilizations } \\
\text { Civil disobedience } \\
\text { Social determinants of terrorism }\end{array}$ \\
\hline Military & $\begin{array}{l}\text { Organizational networks } \\
\text { Command and control } \\
\text { Force on Free }\end{array}$ \\
\hline
\end{tabular}

Unbelievable scenarios for agent based modelling are not just result of the knowledge picked up with multi agent imitations, the convenience of easy to use reproduction phases, more remarkable computer control, and improved awareness methods. A number of exemplar shifts are also predictable. The sociologies are currently meeting a move from poor information to a rich information situation. This permits one to settle or misrepresent models, regulate their limits, and to move to information determined displaying methods (Henderson, Storeygard \& Weil 2012). Furthermore, it will be imaginable to improve the level of detail, precision, and scope of agent-based models by desires of greatness. In the interim, on explanation of the convenience of easy to use regeneration gadgets, the progression times for multi agent renovations will shrink meaningfully.

The operation of techniques from quantifiable material science and the theory of complex outlines to financial information produces an option of moving past informative models towards logical prototypes. The improved information circumstance boosts the insight of the inward influences and notable examples of complex frameworks.

New possible consequences to mine constant information make the chance to change from approximations with a delay, for instance, the outdated methods for determining the overweight national product or the amount of individuals, who have this period's flu virus, towards reliable continuing assessments. Besides, using specific belongings of spreading events in systems, it seems to be even imaginable to achieve two-week predictions (Henderson, Storeygard \& Weil 2012). All the more mostly, reality mining will inspire multi-agent imitations of practical circumstances, the declaration of model limits on the fly, and the fitting pledge of guidance ahead of time symbols. It will similarly desist from dislocating stays and to enlarge the efficiency of substitute reaction events.

Multi-agent imitations will organise approximation based data mining and model-based rebuilding methods. This method goes past hopeful unceasingly information bases, for instance, initial and limit circumstances, parameters, and system qualities into multi-agent re-presentations: it plays out an information determined design salutation and modelling in similar to relevant computer imitations and, therefore, joins the abilities of both policies to attain the ideal exactness and steadiness.

Multi-agent imitations could be explicitly joined with workroom and web tests. Certainty be expressed, the selections of agents in computer imitations could be taken by unpretentious individuals. Unpretentious multi-player onlinegames provide the chance of containing a considerable amount of persons into the examination of composite information and the examination of practical rudimentary decision-making circumstances in effective worlds, which virtually outline future worlds. Like this, agent based imitation practices might be linked for group obtaining and governance applications, to make use of the wisdom of troops (Helbing \& Balietti 2011). For instance, one could inhabit the three-dimensional simulated model of another mall, air terminal, or railroad station, with an exact end goal to learn out, how well the constructions would please its ability, and to figure out which is reinforced by future customers.

The overlapping of genuine and simulated worlds will be a realm of the future. For example, Google Earth and similar virtual depictions of this present realism could be occupied with re-formed persons or genuine ones. Truth be told, persons agreeable to share their GPS guidelines could be verbal to in these worlds precisely, to the level of aspect they like (Helbing $\&$ Balietti 2011). A prolonged reality method would allow persons to share data about their safety, basics, values, and so forth. The degree of data shared might be selected logically or by the kinds of suggestion assistants for example, individuals are obligatory to share secretive data all the more clearly with persons they consider to be their friends. Such extended reality strategies will have the volume to fill in as translator for persons with various vernaculars or social basics, serving them to make themselves more valuable.

Relationship between cars ownership tax and the import tax of cars with the usage of public transport service

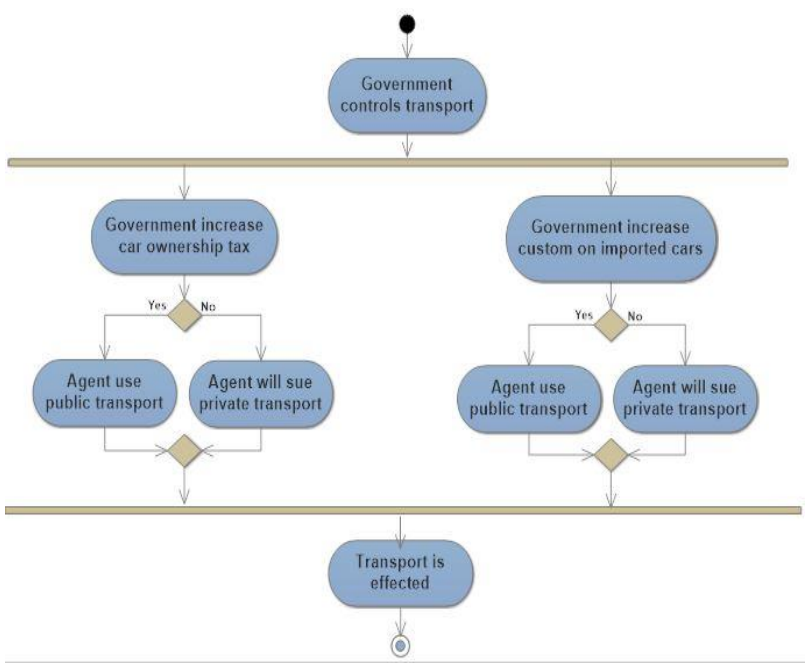

Figure 2: Effects of government control for car ownership tax and customs on imported cars

In the diagram the price elasticity of demand is described as if the government increases the car ownership tax on vehicles, the agents start to use the public transports. As the government charges more and more taxes on the cars owned by people, the 
agents will not be interested in using their own vehicles, so they will switch to public transportation.

Secondly if the government don't increase the tax for the ownership of cars, the agents will continue using their own cars, as it is a better way of travelling with ease.

Another factor will be the custom on the imported cars. If the government increases the custom charge on the imported cars, the agent will start to use either the local cars, or public transport. If the government doesn't add taxes to the imported cars, the cars will not be charged much, then the agents will start to use the imported cars, and the demand for the public transport will be decreased.

\section{Agent-based Transportation Demand Simulation}

The defined work is grounded on agent-based simulation (ABS). ABS is a minute methodology for defining multifarious and dynamic systems. Because of the comprehensive arena of application, ABS is widely held for inquiries of diverse research problems. Henceforth, it is an interdisciplinary method that frequently touches numerous research fields. If $\mathrm{ABS}$ is used for modelling multifarious economic systems, for instance, business and customer model analysis, it is also named agent-based computational economics (ACE). Particularly because of its foundation on separate decisionmaking, ABS is also used in the field of transportation demand simulation.

\subsection{Simulation Methodology}

The implemented simulation method is grounded on the open source instrument MATSim. This instrument for agent based transportation demand imitation was established, enhanced and recognized as an appropriate instrument for research. To observe the TDM special effects, the stimulation approach is modified in different regions.

At the start, important input figures need to be converted into a data layout that can be used by MATSim. In the historical years, investigators recognised movement chains of agents as a significant portion of public transport simulation. The essential data is often collected by surveys. In this effort additional agent-based simulation of individual everyday procedures is used, which is founded on the instrument SeSAm2, as a data input. The network data of the experiential German city area of Aachen was transformed this way. After modifying the populace and network statistics of Aachen, essential events and agent plans were constituted (Helbing \& Balietti 2011). Then, each agent which was chunk of the generally population database was modelled, dependent on the quantitative survey outcomes. The precise sensitivity of each person towards different inducements was assessed, and, as significance, modelled in the agent database. This was comprehended by adding precise agent qualities. During the imitation, normally agents evaluate their happening through a counting function. The default counting function by MATSim does not evaluate the transport mode clearly, and the fresh designed agent qualities are not merged into this process either.

Hereafter, the counting function was stretched to integrate incentive approximation, which is modelled as an individual agent quality, into the complete agent counting. Changes were grounded on the Leg Scoring. In case of enticements, agents assessed the plan dependent on their necessities towards the assured enticement. Successively, the simulation contained of packing applicable network and people data (Gardner 1970). Later, agent tactics were directed in the network. Thus, dependent on events, e.g. traffic mobbing, agent plans jointly prejudiced each other. Then, agent plans were judged through the overextended counting function. After scoring, agent plans were enhanced, reliant on the given policies during the arrangement phase. This procedure defines one repetition; in which lower counted plans are released in favour of better predictable plans. This repetition loop aids as a rising decision basis for agents to enhance their behaviour. The simulation was achieved in up to fifty repetitions. Lastly, the results were matched with the survey data to legalize the prototypical parameters.

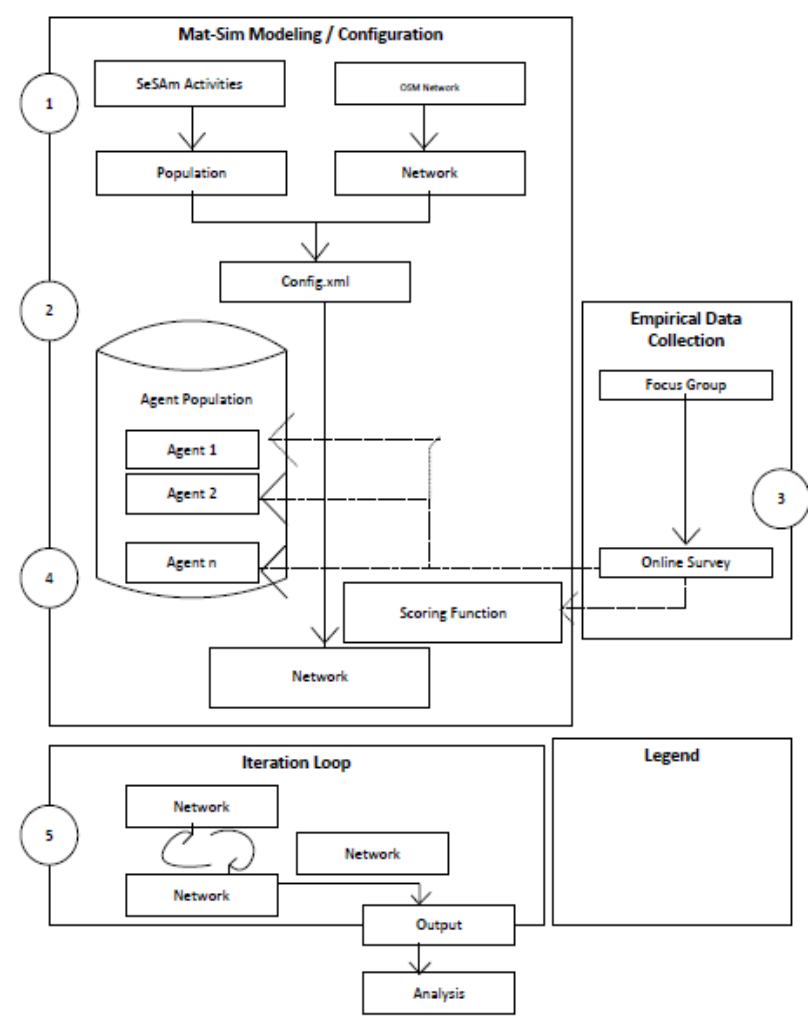

Figure 3: Mat-Sim Modeling / Configuration (Source: MATSIM)

\subsection{Simulation Results}

After the authentication process, imitation results portrayed the demand effects analogous to the quantitative survey data. All examined incentives presented applicable effects: the agent's choice of carpooling enlarged, while the use of own car reduced. These were also the only two resources of conveyance for which the portions changed melodramatically when the incentives were familiarized, the practice of bike, community transport and walking barely changed at all. So, the last will not be argued here in detail. The simulation additionally presented that while only one enticement was used, importance parking had the smallest effect compared to the situation with no enticements. Regarding the mixture of enticements, it can be realised that the more enticements are presented at the same time, the better the portion of carpooling.

This detailed method examined people's tendency concerning certain incentives, which permitted to simulate a degree of comparison and combination. Results revealed that the 
incentives selected for this simulation merely had an influence for car users in the manner that they would be more eager to contribute in carpooling. The point that bike practice and walking did not alter significantly when incentives were familiarized could be owing to the nature of these two styles: they can be used adaptably, do not charge anything and no coupon is needed for them, so the enthusiasm to use them might be different as of for instance car and public transport. Above all, this work validates the hypothesis that agent-based demand simulation is an appropriate mechanism to observe the special effects of TDM measures and enticements in urban transport in contrast and combination.

Likened to recognized TDM assessments, agent-based demand simulation examines the connections of large numbers of objects in composite transportation networks. Besides, daily practices and situational special effects like traffic mobbing can be combined into investigations. This enhances an additional value for planning procedures. However, there are some disadvantages associated with it. First, the descriptive power of ABS methods is considerably reliant on the fundamental data quality (Jennings 2000). To authenticate an ABS in this technical field, an extensive examination under truthful circumstances is more appropriate than an online inspection. In addition, demand simulation becomes more complex in the area of monetary incentives or guidelines. Even then, it is essentially possible to prepare agents with finances about agent modeling, the focus groups presented that contributors have difficulties to stipulate a certain mobility budget. Furthermore, focus groups specified the individual necessities regarding specific measures. In fact, findings like these emphasize the valued involvement of focus group studies previously in the start of modeling and simulation procedures of human behavior and connections.
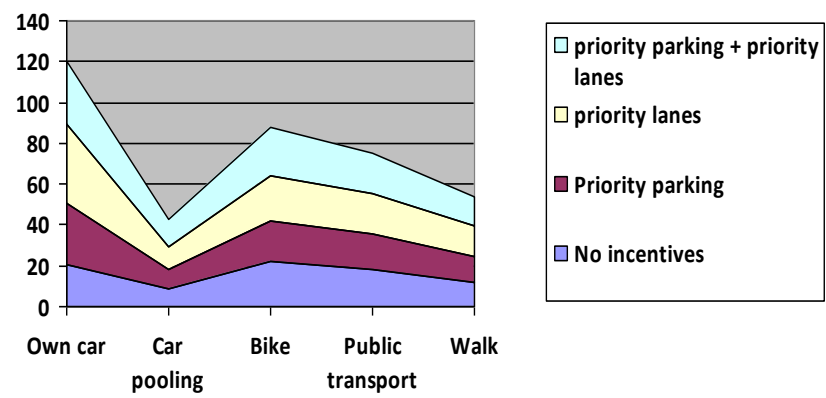

Figure 4: Agent priorities in graph (Source: Jennings, 2000)

\section{Agent-Based Modeling for Route Choice Behaviors}

The benefits and strength of integrating ABMS to observe route choice behavior of travelers, rather than the outdated route choice models, comprise the ability to:

- Capture a specific traveler's coherent and illogical conduct and inclinations that are tough to calculate or measure in the outdated route choice models.

- Identify and ponder that travelers have dissimilar socioeconomic possessions, travel practices, partialities, way of reaction to the in route information, etc., and thus show heterogeneity.

- Capture the communication effects and joint performance that stalk from travelers' heterogeneity.

- Identify and ruminate that travelers may have imperfect information about issues such as traffic circumstances, occasions, and climate conditions; hereafter, ABMS captures the haziness of driver behavior, not like separate optimal models that assume drivers are always balanced with faultless admittance to full information.

- Allow travelers' choice and information to be efficient on a real-time base, rather than on a day-today base.

- Articulate the mechanism of travelers' composite decision making procedure.

- Forecast travelers' sensible reply to real-time in route information or related unanticipated spur forced in the environment.

- Probably observe developing behavior as an inducement to a new environment system.

Relationship between the prices of the public transport with the usage of the private transport and public transport

The diagram reflects the Elasticity of the Demand. The elasticity of the demand of the public transport is also dependent on the maximum prices of the transport. If the government will increase the maximum prices of the public transport, then the agent will not be able to afford the public transport at all, in this case the agent prefers to use the private cars. So there is an inverse relationship between the maximum prices of the public transport, and the prices of the transport.

If the government will decrease the maximum prices of the public transport, the agent will start to use the public transport, and the use of private automobiles will be reduced.

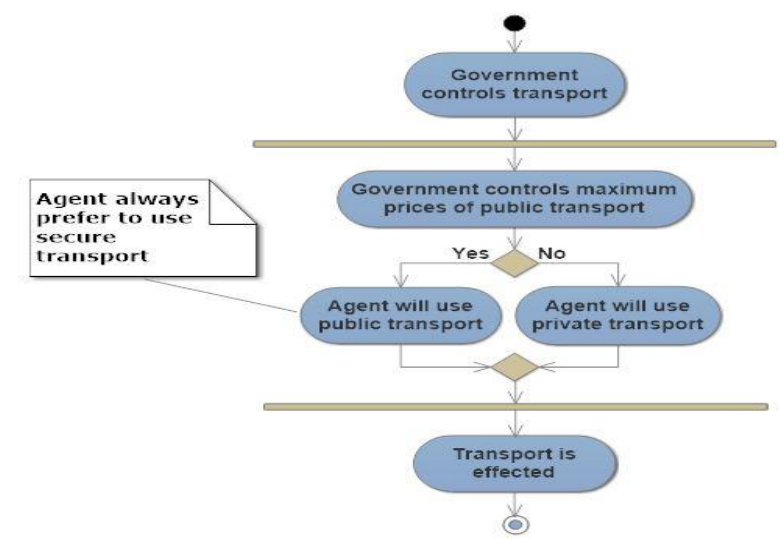

Figure 5: Effects of governmental controls of public transport prices

Relationship between the prices of the fuel and the parking fee with the usage of the public transport

The Cross price elasticity is also a factor between the prices of the fuel, the prices of the parking and the usage of the transport.

There are two main cases:

- If the government increases the prices of the fuel, it will cause the agent to use the public transport. The increase in the fuel price will affect the usage of private cars. This will eventually change the preference of people to public transport.

- Secondly the parking prices also have an effect on the usage of private owned cars. If the parking 
charges are set higher in the areas, the usage of private cars will stop. They will prefer to use the public transport as it doesn't require any parking fee.

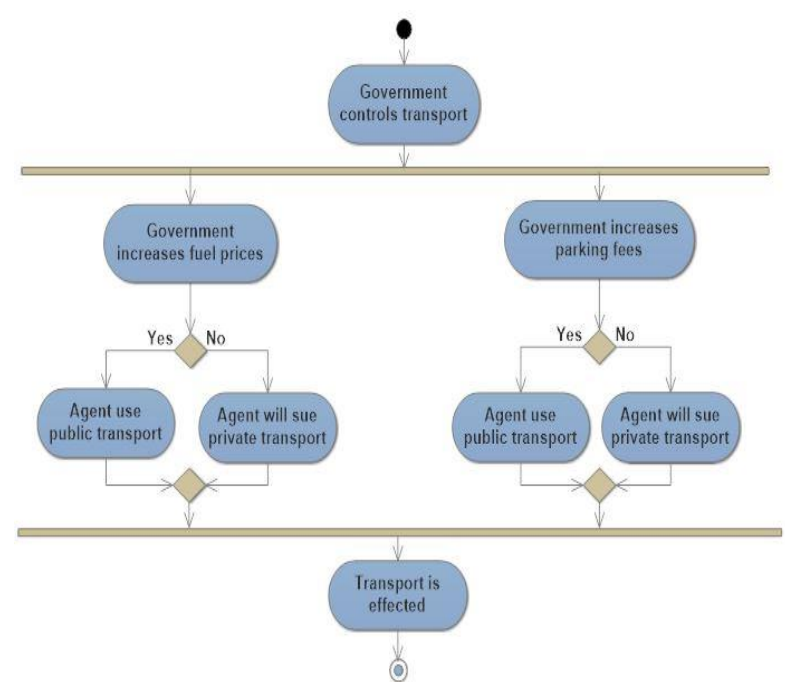

Figure 6: effects of government control of fuel and parking fees

Factors that show negative and positive effect on public transport

There are certain factors discussed here in detail that can influence the decision of using either the public transport or private:

The fixed number of stops and destinations can help in increasing the usage of the public transport. If the stops and destinations are increased, people will prefer to use the public transport, so they can travel to more and more places through the public transport.

- If the timetable for the transport is maintained properly, then people will use the public transport for travelling. If proper information about the timings of the public transport vehicles is disseminated, people will use these vehicles for travelling.

- $\quad$ If maintaining the above factors increase the usage of the public transport, then there is a need from the government increase the supply of the public transport. If the usage of public transport increases, there will be a need to increase the number of vehicles also. In this way government will need to increase the supply of the public transport.

To maintain the public transport, the information about the public transport should be easily available. The information should be displayed on the public places so that people may get the proper information about the public transport vehicles, routes and timings.

Finally, the public transport usage can be increased by increasing the reliability of the transport which means the transport quality should be increase. The buses should be clean and comfortable and also on time. The comfort with time and space will influence people to use the public transport more.

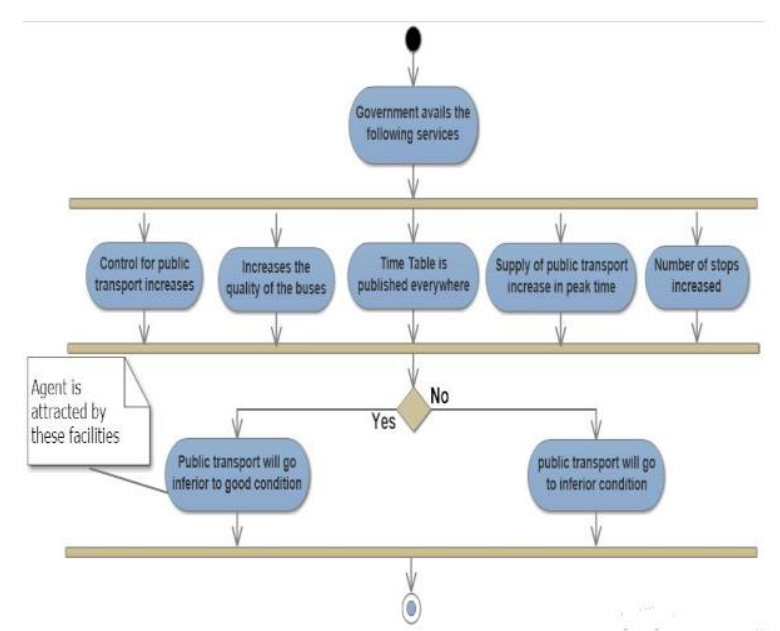

Figure 7: Effects of government avails for some services

\section{Positive steps to increase the usage of public transport}

The usage of public transport also increases once the government starts subsidising the public transport. It decreases the overall cost of usage to the people. This in turn increases the chance of usage because of affordability. However, if the government is not subsidizing the public transport, due to increased fuel prices, the affordability of public transportation is reduced which influences people to use the private transportation more.

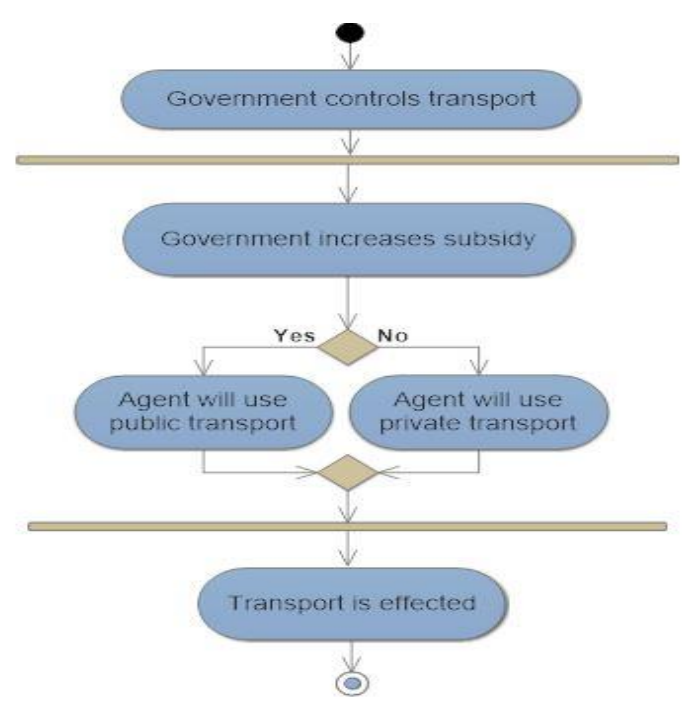

Figure 8: Effects of government controls for subsidy

\section{Safety measures and its effect on public transport}

Below is a safety paradigm for passengers. Safety in the cars is lesser as compared to safety in the bus. The usage of public transport is much safer than the usage of personal cars. Moreover, there are certain facilities that the public transport offers. Like, picking up from a designated spot and dropping at your destination without the strain of driving, stress of traffic 

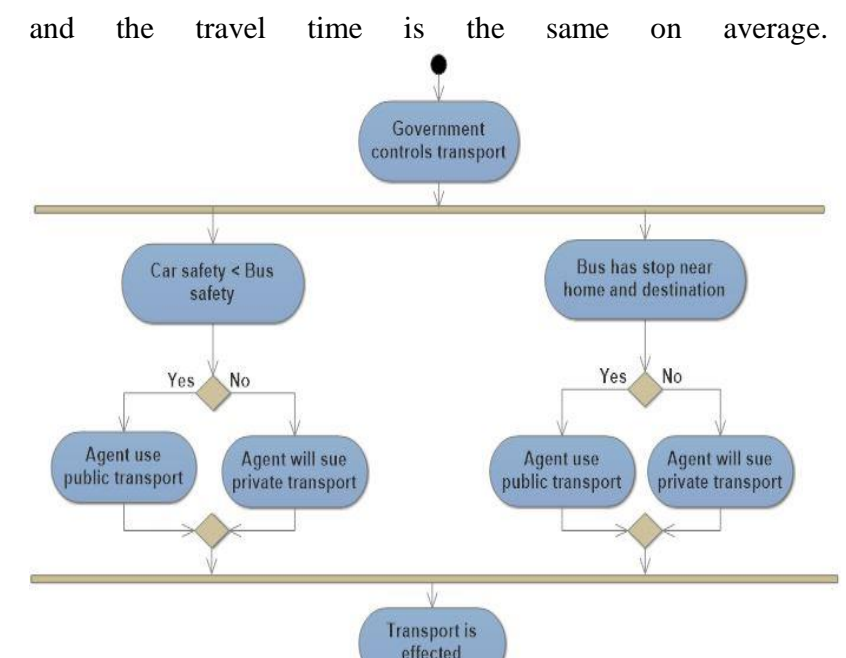

effected

e

Figure 9: Government controls for car safety and stops

\section{Conclusion}

Agent-based modelling has been the keystone of prosperous modern networks. In this spirit, the importance of the model cannot be overlooked. The demand for such models is more, predominantly in the field of the transport models and networks, as the number of automobiles on the roads is increasing by the year.

Agent-based models have been used to mimic almost every feature of the economic, social, and technical fields. One such application is for the transport model and related human performance that depend upon one or more expectations. Human behaviour, though, cannot be controlled by a set of guidelines. However, this model will deliver a significant landmark in human evolution towards better extrapolative measures of the infrastructures and other application but will always have a nonexistence important humanistic element: inimitable cognitive processes of the persons. In this respect, the internet of things (IoT) outline will be critical in successful and effective enactment of the agent based modelling methods.

\section{References}

1. Samuelson, DA, Macal, C.M.: Agent-Based Simulation Comes of Age. OR/MS Today (2006)

2. Bonabesau, E.: Proceedings of The National Academy of Sciences 99 (2002)

3. Macal, C.M., North, M.J.: Proceedings of The 38Th Conference on Winter Simulation, 1st ed. Winter Simulation Conference (2006)

4. Helbing, D.: Competence Center Coping With Crises in Complex Socio-Economic Systems CCSS Working Paper Series (n.d.).

5. Helbing, D., Balietti, S.: The European Physical Journal Special Topics 195 (2011)

6. Foster, I: Nature 440 (2006)

7. Epstein, J.: Down's Syndrome: Critical Genes in a Critical Region. Nature 460 (2009)

8. Epstein, J., Axtell, R.: Growing Artificial Societiess. Brookings Inst. Press, Washington DC (2007)

9. Gardner, M.: Scientific American. Mathematical Association of America; Washington DC 223 (1970)

10. Jennings, N: On agent-based Software Engineering. Artificial Intelligence, vol. 117, no.2, pp. 277-296 (2000)

11. Henderson, J., Storeygard, A., Weil, D.: Measuring Economic Growth from Outer Space. American Economic Review, vol.102, no.2, pp. 994-1028 (2012)

12. U.S. Department of Transportation, A Primer for AgentBased Simulation and Modelling In Transportation Applications. Federal Highway Authority (FHWA) (2013)

13. Agent-based Transportation Demand Management Demand. Effects of Reserved Parking Space and Priority Lanes in Comparison and Combination (2013)

14. A Primer for Agent-Based Simulation and Modeling in Transportation Applications

15. U.S. Department of Transportation, A Primer For AgentBased Simulation And Modeling In Transportation Applications, Federal Highway Authority (FHWA), 2013. 